\title{
New techniques
}

\section{Imaging the spine}

\author{
M Gibson, A Al-Kutoubi
}

Summary

This article on neuroradiology concerns the imaging of the spine. The imaging modalities are discussed, and some basic imaging strategies are outlined.

Keywords: imaging, spine

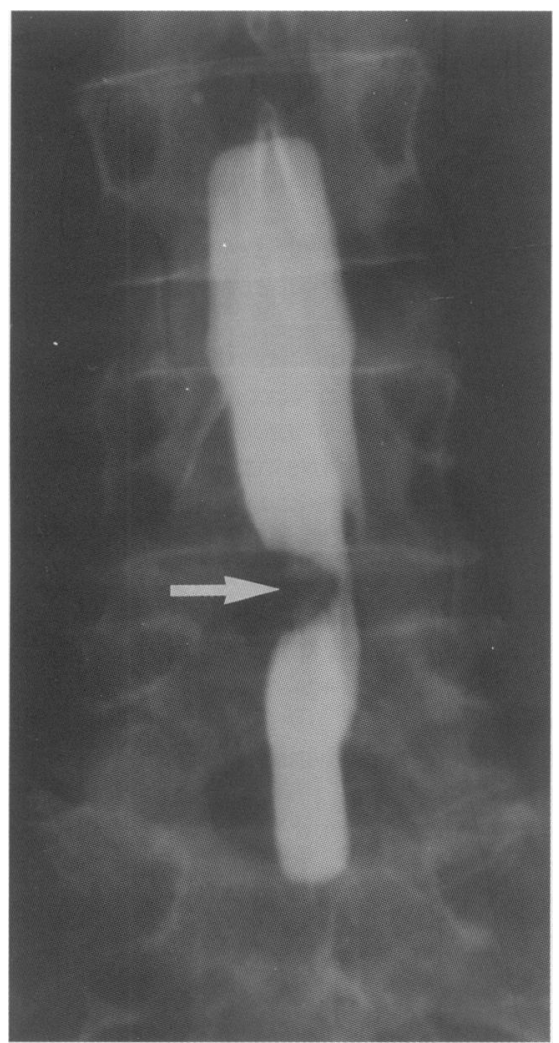

Figure 1 Myelogram of the lumbar spine. A large disc protusion (arrow) is impinging on the thecal sac (containing white contrast)

\begin{tabular}{|l|}
\hline Imaging modalities for the \\
spine \\
\hline - plain X-rays \\
- computed tomography \\
- magnetic resonance imaging \\
- myelography \\
- discography \\
- facet joint arthrography \\
\end{tabular}

Department of Radiology, St Mary's Hospital, London W2, UK

M Gibson

A Al-Kutoubi

Accepted 16 October 1995

\section{Imaging modalities}

\section{PLAIN RADIOGRAPHY}

The standard frontal and lateral films are useful in detecting bony abnormality, alignment and fractures. Obviously they do not demonstrate the soft tissues of the spine (eg, disc, cord or ligaments) and are therefore of limited use.

\section{COMPUTED TOMOGRAPHY (CT)}

Usually the patient lies in the CT scanner. The scout view is obtained and then selected levels are scanned axially. If the slices are thin enough, good quality sagittal reformation can be performed. In trauma, CT is useful in detecting fractures and bony fragments. It is of limited sensitivity for imaging the soft tissues and is therefore sometimes performed after myelography (CT myelogram). However, in most centres magnetic resonance imaging (MRI) is preferred for imaging the soft tissues. Occasionally intravenous contrast is given. In the postoperative patient this is usually to distinguish scarring from the normally enhancing dura and epidural tissue.

\section{MAGNETIC RESONANCE IMAGING}

In many circumstances MRI is now the modality of choice for spinal imaging. Usually T1- and T2-weighted images are obtained in the axial and sagittal planes. The soft tissue contrast and particularly cerebrospinal fluid/spinal cord distinction is good. This enables excellent visualisation of disc, ligaments, spinal cord and nerve roots. The long scan times mean that motion artefacts can degrade the images considerably. However, quicker sequences are being developed to overcome this. MRI cannot be performed in the presence of certain contraindications ${ }^{1}$ or in patients who are claustrophobic or too heavy for the scanner. In these circumstances, either myelography or CT are performed.

\section{MYELOGRAPHY}

Myelography has largely been superceded by MRI. It is an invasive procedure that necessitates a lumbar puncture (or even occasionally a lateral cervical puncture) and installation of non-ionic water-soluble contrast media into the subarachnoid space. There is strict adherence to a maximum dose of contrast media. Cord compression, impingement and nerve root compression can be demonstrated (figure 1). Side-effects include headache, fits and other contrast reactions. Infections and implantation dermoids are rare.

\section{ANGIOGRAPHY}

Spinal angiography is only rarely performed. If an arteriovenous malformation is suspected from the MRI or myelogram then angiography can be used to confirm the diagnosis (figure 2) and possibly to perform therapeutic embolisation. The spinal cord has a variable vascular anatomy. The spinal arteries usually arise from the intercostal and lumbar arteries. They can be selectively catheterised from a standard femoral approach. There is definite morbidity including ischaemia of the cord.

\section{DISCOGRAPHY}

This is an uncommon investigation of back pain rather than cord or nerve root symptoms. A spinal needle is introduced from the posterolateral approach (avoiding the spinal cord) into the annulus of the disc (usually lumbar) under fluoroscopic control. Contrast is introduced to demonstrate any herniation (figure 3). The sensation at the time of injection is compared to the original symptoms in an attempt to localise the cause of the symptoms. This technique is also the preliminary to chemical nucleolysis when chymopapain is injected into the annulus.

\section{FACET JOINT ARTHROGRAPHY}

Under CT or fluoroscopic control a spinal needle is introduced into the facet joint. Contrast can be injected to confirm position in the joint. At the same time 


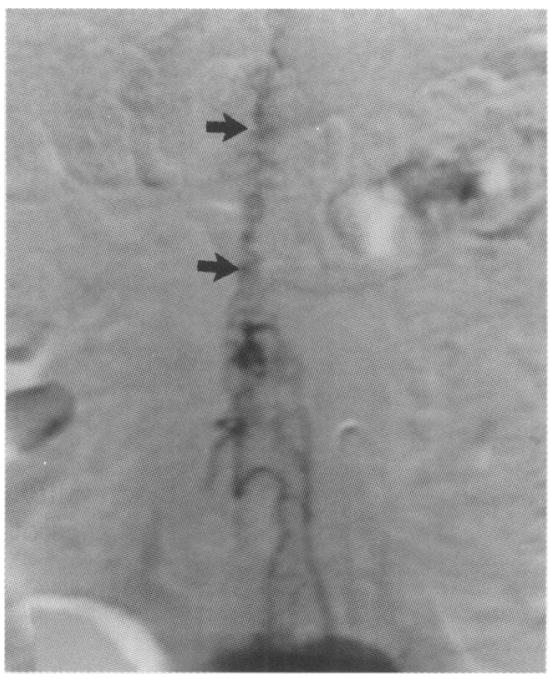

Figure 2 Spinal angiogram. A line of abnormal tortuous vessels indicate an arteriovenous malformation (arrows)

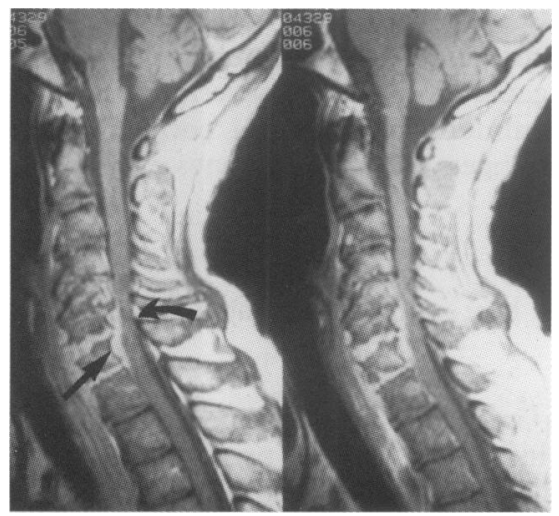

Figure 5 MRI of the cervical spine. TIweighted image post-contrast. There is bone destruction and enhancing soft tissue in the epidural space (straight arrow) that is causing compression of the spinal cord (curved arrow). This is an epidural abscess and was due to tuberculosis

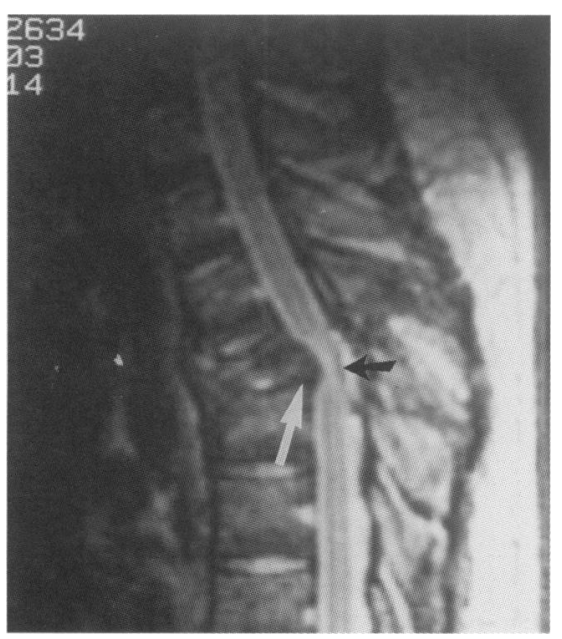

Figure 6 MRI of the thoracic spine. T2weighted image. In this case of clinical cord compression following trauma there is a crush fracture of the vertebral body with a traumatic disc herniation (white arrow) causing compression of the thoracic cord (black arrow)

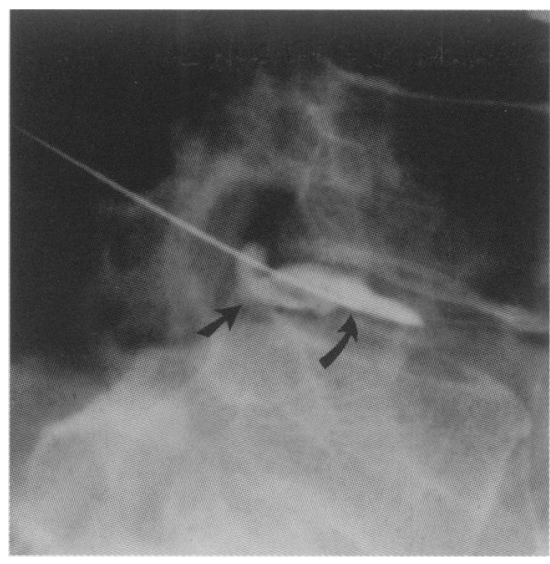

Figure 3 Discogram. A needle has been introduced into the annulus of the disc (curved arrow). Contrast fills the annulus and demonstrates a large disc protusion (straight arrow)

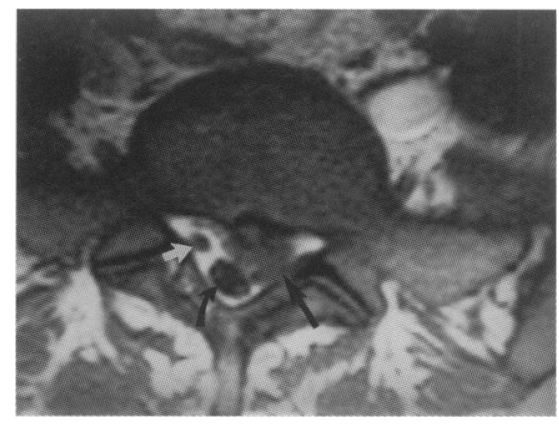

Figure 4 CT scan of the lumbar spine. Axial slice. There is a large disc protrusion (straight black arrow) compressing and displacing the thecal sac (curved black arrow) The normal nerve root on the right is shown (white arrow). On the left the nerve root is compressed by the disc protusion

steroids and local anaesthetic can be injected to treat back pain thought to be arising from the facet joints.

\section{NUCLEAR MEDICINE}

Abnormal activity within the vertebrae or other parts of the skeleton can be detected on an isotope bone scan. This test is sensitive but of low specificity as a variety of benign and malignant conditions can result in abnormal uptake of the isotope. It is primarily used in the assessment of metastatic disease.

\section{Imaging strategies}

The following possible strategies are divided into those for acute and chronic presentations. They are not intended to be dogmatic and obviously depend upon local availability.

\section{ACUTE PRESENTATIONS}

\section{Back pain}

This usually resolves with conservative treatment and plain films are reserved for cases of trauma or symptoms lasting longer than six weeks.

\section{Nerve root irritation}

Again this often resolves with conservative treatment. If symptoms are severe or have associated features of cord compression then MRI is performed. If unavailable, myelography or CT (figure 4) are alternatives.

\section{Spinal cord symptoms}

These symptoms are usually assumed to be due to cord compression until proven otherwise. They are an indication for urgent investigation usually with MRI if available (figure 5). If unavailable, myelography and sometimes CT myelography is indicated. If there has been trauma MRI is the investigation of choice (figure 6). Additional CT can be useful in imaging the bony spine.

\section{CHRONIC PRESENTATIONS}

\section{Pain}

The failure of pain to respond to conservative treatment warrants plain films to exclude any bony abnormality. If the clinical features indicate the need for further investigations then MRI is preferred. Obviously clinical screening of these patients is essential in such a common clinical condition. If symptoms are referrable to the facet joints then arthrography and steroid injection may be warranted. Isotope studies can be performed if there is suspicion of metastatic bone disease (figure 7).

\section{Nerve root irritation}

Sciatica is often treated conservatively; if clinically indicated, investigation is 


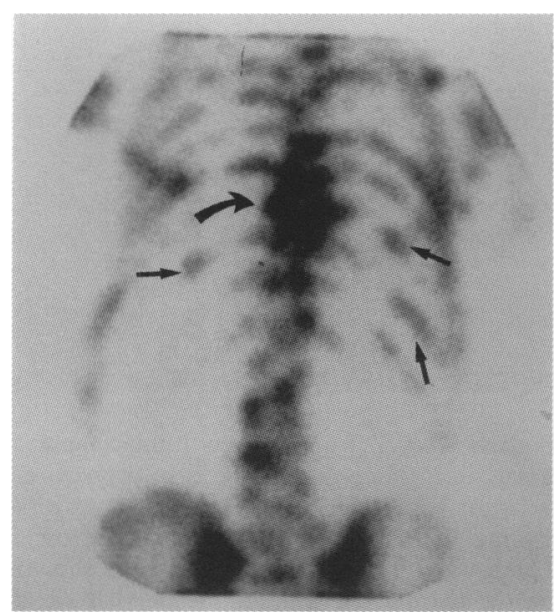

Figure 7 Isotope bone scan. There are multiple metastatic deposits throughout the skeleton including the spine (curved arrow) and ribs (straight arrows)

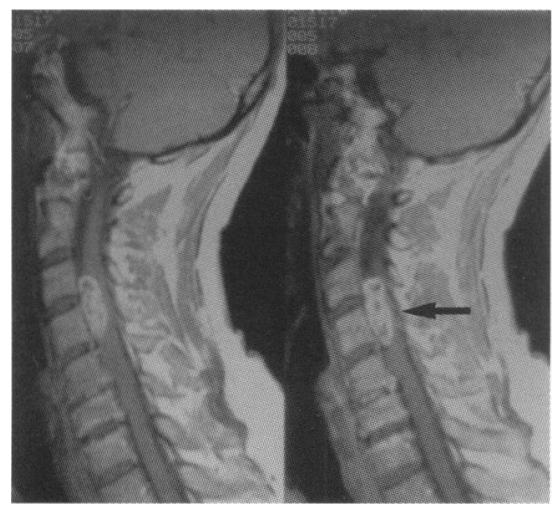

Figure 10 MRI of the cervical spine. TIweighted images post-contrast. There is an enhancing mass within the cervical cord (arrow) that proved to be a benign neurofibroma

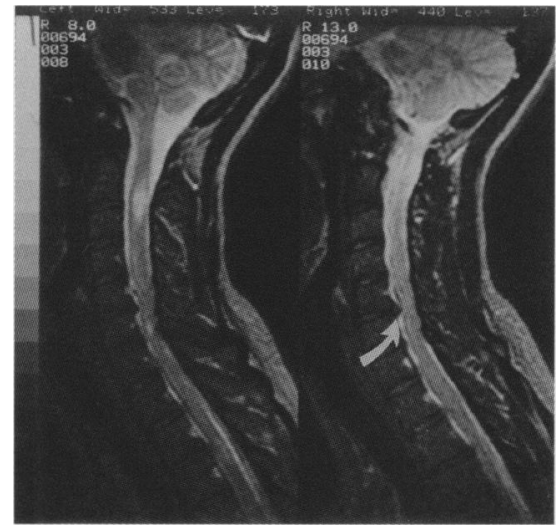

Figure 8 MRI of the cervical spine. T2weighted images. There a disc protusion (arrow) impinging on the cord

usually with MRI. In the arm symptoms are often due to degeneration of the cervical spine. Plain films will detect degeneration and its severity (although degenerative change on plain films is common and may not correlate with symptoms). MRI can be used to detect impingement of osteophytes or discs on nerve roots (figure 8).

Chronic cord compression

As with the acute presentation MRI is the best modality available (figure 9).

Other cord symptoms

Noncomprehensive pathologies such as intrinsic cord lesions (primary or secondary tumours), demyelination, or inflammation can present with cord symptoms. MRI is the imaging modality of choice (figure 10). CT or myelography can be used if MRI is unavailable.

1 Gibson M, Cook G, Al-Kutoubi A. Intracranial imaging. Postgrad Med f 1996; 72: 386-90.

\section{.}

Figure 9 MRI of the cervical spine. TIwest contrast. This metavertebral body (curved arrow). It is displacing the cord posteriorly and extending into the prevertebral soft tissues (straight arrow) 